\title{
How Research on Microbiomes is Changing Biology: A Discussion on the Concept of the Organism
}

\author{
Adrian Stencel $^{1} \cdot$ Agnieszka M. Proszewska $^{1,2}$
}

Published online: 31 October 2017

(C) The Author(s) 2017. This article is an open access publication

\begin{abstract}
Multicellular organisms contain numerous symbiotic microorganisms, collectively called microbiomes. Recently, microbiomic research has shown that these microorganisms are responsible for the proper functioning of many of the systems (digestive, immune, nervous, etc.) of multicellular organisms. This has inclined some scholars to argue that it is about time to reconceptualise the organism and to develop a concept that would place the greatest emphasis on the vital role of microorganisms in the life of plants and animals. We believe that, unfortunately, there is a problem with this suggestion, since there is no such thing as a universal concept of the organism which could constitute a basis for all biological sciences. Rather, the opposite is true: numerous alternative definitions exist. Therefore, comprehending how microbiomics is changing our understanding of organisms may be a very complex matter. In this paper we will demonstrate that this pluralism proves that claims about a change in our understanding of organisms can be treated as both true and untrue. Mainly, we assert that the existing concepts differ substantially, and that only some of them have to be reconsidered in order to incorporate the discoveries of microbiomics, while others are already flexible enough to do so. Taking into account the plurality of conceptualisations within different branches of modern biology, we will conduct our discussion using the developmental and the cooperation-conflict concepts of the organism. Then we will explain our results by referring to the recent philosophical debate on the nature of the concept of the organism within biology.
\end{abstract}

Keywords Pluralism · Microbiome $\cdot$ Organism · Philosophy $\cdot$ Biology

Adrian Stencel

adstencel@gmail.com

1 Faculty of Philosophy, Jagiellonian University, ul. Gołębia 24, 31-007 Kraków, Poland

2 Faculty of Physics, Astronomy and Applied Computer Science, Jagiellonian University, ul. prof. Stanisława Łojasiewicza 11, 30-348 Kraków, Poland 


\section{Introduction}

Multicellular organisms, which we will refer to here as macrobes, following O'Malley and Dupre (2007), contain numerous symbiotic microorganisms, collectively called microbiomes (Lederberg 2000; Zilber-Rosenberg and Rosenberg 2008), within their bodies. It is worth noting that the number of microorganisms forming a microbiome is often equal to the number of cells that make up the macrobe's body (Sender et al. 2016). Furthermore, the role of symbiotic entities in the functioning of macrobes has recently been the object of intensive research, which has led to many interesting discoveries: for example, that the presence of some microorganisms is necessary for the proper development of the immune system (Mazmanian et al. 2005), functioning of the nervous system (Carabotti et al. 2015), and digestion of necessary nutrients (Ley et al. 2006), and that some microbes may even be the agents responsible for the evolution of new species (Sharon et al. 2010; Brucker and Bordenstein 2013). Due to this extraordinary role, some biologists have even suggested that microorganisms living in symbiosis in and on human bodies should be collectively regarded as a new organ (Baquero and Nombela 2012; Clarke et al. 2014).

Those discoveries have led some to suggest that, given the essential role of microorganisms in the functionality of macrobes, microbiomic research is changing our understanding of the concept of the organism ${ }^{1}$ within biology, and that it is high time to reconsider this concept so as to include symbiotic microorganisms (Sleator 2010; Gilbert et al. 2012; McFall-Ngai et al. 2013). For instance, Sleator (2010) stated: 'For years our traditional view of 'self' was restricted to our own bodies, composed of eukaryote cells encoded by our genome. However, in the era of omic technologies and systems biology, this view now extends beyond the traditional limitations of our own core being to include our resident microbial communities.' McFall-Ngai et al. (2013) argued in the same vein that: 'These new data are demanding a re-examination of the very concepts of what constitutes a genome, a population, an environment and an organism'. Obviously, there is something important going on in biology, as microbiomics calls many standard views into question. But are these discoveries really so momentous that we can no longer think about organisms in the old way, as these scholars suggest?

We believe that we should really be very careful when making such statements, because there is no such thing as a single universal concept of the organism which could constitute a basis for all biological sciences. There is no single 'old way' of thinking about organisms. Rather, in biology, numerous alternative concepts of the organism exist (Pepper and Herron 2008; Queller and Strasmann 2009; Clarke 2010). Therefore, understanding how microbiomics is changing our understanding of organisms may not be so easy. How should we deal with this situation? We can envision two plausible scenarios. According to the first, these different conceptualisations, though they may differ substantially, all respond to the research on microbiomes in the same way. Each will have to be reconsidered in order to incorporate ongoing discoveries. If so, then research on microbiomes may change the way organisms are understood within biology. This point was made by Gilbert et al. (2012), who argued that all concepts of organisms are called into question by recent discoveries in the field of microbiomics. According to the second scenario, the various conceptualisations respond differently to ongoing discoveries. If true, this may undermine the statement that

\footnotetext{
1 The meaning of the words organism and individual has never been clear in biology. Therefore, we use them interchangeably following the majority of scholars (see Pepper and Herron 2008; Gilbert et al. 2012; Clarke 2013). To refer to different definitions of organism/individual, we will use adjectives: for example, genetic organism/individual, immunological organism/individual, etc.
} 
biology is undergoing change in its understanding of organisms, since one concept of the organism may have to be rejected, while another concept can easily deal with the results of microbiomics research. This would ensure that changes in the way of thinking about organisms are local, i.e. mostly constrained to certain concepts which cannot manage to incorporate the ongoing discoveries.

In this paper we will argue in favour of the latter scenario. Mainly, we will show that even though research on microbiomes is spectacular, it does not influence every concept of the organism in the same way. To prove this point, in the next section, we will analyse two concepts of the organism to show that they respond differently to microbiome research. Then we will explain our results by referring to the recent philosophical debate on the nature of the concept of the organism within biology.

\section{Microbiomics and Concepts of the Organism}

It would not be an exaggeration to say that the concept of the organism has been a centre of interest in recent years for both biologists and philosophers. A great deal of work has been done on answering the question 'What is an organism?' (Clarke 2010, 2013; Queller and Strasmann 2009, 2016). This might be surprising at first glance, because we all intuitively think we know what organisms really are: a kind of functional cohesive whole. Cats are organisms, stones are not. Apes are organisms, a river is not. The motivation for this work is, however, entirely justified and derives from one basic fact: namely, that it is sometimes very hard to decide whether a given object is an autonomous organism or merely a part of another organism (Wilson 2000; Pepper and Herron 2008; Clarke 2010). Of course, this is not at all problematic when we deal with species like cats; there are good reasons to say that a cat's heart is just a part of the cat. However, there are examples that have been puzzling scientists for decades. For instance, should we consider an entire ant colony to be one big organism, or should we, perhaps, grant the status of an organism to each ant?

In light of such problematic cases, scholars have developed many different approaches toward defining organisms. For instance, we have concepts, based on genetics, that individuate organisms by suggesting that an organism is something that possesses a unique genotype (see Santelices 1999). Other scholars focus on the process of reproduction, which is supposed to mark the distinction between individuals and non-individuals, by arguing that an organism is something that comes from a fertilised egg (see for instance Janzen 1977; Slack 2005, p. 6). Still other thinkers argue that organisms are objects capable of evolution via natural selection (see Clarke 2013; Godfrey-Smith 2009). And these three examples are just the tip of the iceberg. For instance, Clarke (2010) listed 13 definitions of the organism. Due to lack of space, we obviously cannot analyse all of them here; therefore, we have chosen two, as sufficient to make our point clearly: the developmental concept of the organism and the cooperation-conflict concept of the organism. Our choice is motivated by the fact the former is more concrete, the latter more abstract. Therefore, we suppose that the latter, due to its abstract nature, may be elastic enough to incorporate the ongoing discoveries, while the former may be too narrow to do so.

\subsection{The Developmental Concept of the Organism}

We'll start our analysis with a more concrete and popular approach to defining organisms, one which Gilbert et al. (2012) called the developmental concept of the organism. 
According to this concept, organisms are not fixed units but dynamic entities which undergo a process of development from simple to more complex beings, such as those we observe every day, e.g. cats or apes. Due to the developmental nature of organisms, we can identify a point in space and time as the beginning of an organism and thus can distinguish one organism from another. Usually this point is the fertilisation of an egg by sperm, because this initiates a series of processes leading to the development of a mature, unique organism that possesses sufficient biochemical machinery to produce more eggs and sperm. Or, as Gilbert et al. (2012) put it more poetically, 'the individual animal proposed here is understood to be that which proceeds from ovum to ovum'. This way of thinking about organisms is very popular among not only laymen (as many people believe that new organisms, like members of our own species, start with the fertilisation of an egg by sperm), but scholars too, as it is one of many ways of defining organisms (for an overview, see Pepper and Herron 2008; Clarke 2010). For instance, Slack (2005, p. 6) writes, '[d]evelopment happens most obviously in the course of embryonic development as the fertilised egg develops into a complete organism', and Moore et al. (2016, p. 1) state that '[h] uman development begins at fertilisation when an oocyte (ovum) from a female is fertilised by a sperm (spermatozoon) from a male. Development involves many changes that transform a single cell, the zygote, into a multicellular human being.' This concept of the organism seems intuitive and easy to grasp; it may also seem to define organisms as entities that develop from a fertilised egg. However, we believe that the foundations of this concept are considerably more profound than they seem at first glance. Indeed, in our opinion, the concept is essentially much broader. Therefore, we have to examine its foundations in order to understand whether microbiomics undermines them. We believe that two basic foundations of the developmental concept of organisms can be distinguished.

Let's start by discussing the first. To frame the discussion clearly, however, we should first try to answer a simple question: what kind of observation led to the formulation of the developmental concept of the organism? It should seem obvious that the idea of defining the organism in this way derived from observations of how certain paradigms of organisms, such as cats, apes, or humans, function and develop. The first thing that catches our eye is that these organisms are built of millions of cells that are actively engaged in maintaining the functional coherence and structure of the whole. In other words, they are responsible for processing and assimilating external resources which enable the whole to persist over time. For instance, a cat is made of cells that are well suited, by virtue of division of labour (movement, digestion, etc.), to perform various actions in order to keep the cat's overall structure and functionality undisturbed. Another observation is that the presence of these cells follows from the successive divisions of a zygote. Indeed, the idea behind the developmental concept of organisms depends on the empirical fact that in many species the cells that actively maintain the structure and functionality (such as the digestive or nervous system) of the whole come from a zygote. The emerging concept is then based on the conjecture that an organism is simply a set of cells that develops in the course of the zygote's divisions and that occupies a place within a complex network of various interactions.

Thus, we know which observations lie behind the origin of the developmental concept of the organism. Can we now, from these observations, deduce the foundations of this concept? If we were to take these observations to a higher level of abstraction, we would say that the foundations are that: (1) organisms are functionally integrated units that actively maintain their structures, and (2) they are composed of elements linked by common recent descent. In our opinion, according to the developmental concept, 
organisms are units that attain their full functionality through development from a certain starting point. Indeed, the real idea behind this concept is that there must be a certain point from which all functional elements of the organism 'bloom'. Usually this is a zygote, as many commonly studied species come into existence due to divisions of a fertilised egg, as in the case of apes, cats, etc. However, we don't see fertilisation of an egg as mandatory. This is because functional wholes which actively maintain their structures can also come into existence by starting from a different recent ancestor-for example, from parthenogenesis, which is the process of development of an individual from an unfertilised egg, producing genetically similar but functionally independent aphids, or from a simple split of a human zygote leading to the development of two functionally independent monozygotic twins.

In spite of these examples, we believe that our interpretation of the developmental concept of organism is correct, because it takes the two observations that lie behind the origin of this concept (as mentioned above) into account and extracts from them what, in our opinion, constitutes their foundations. We affirm that a group of elements, in order to be considered a developmental individual, must be functionally integrated and linked by common recent descent (which may, but need not, involve a fertilised egg). This is the interpretation of the developmental concept of the organism that we will work with here. ${ }^{2}$

Of course, cells linked by common descent (e.g. by coming from a zygote) are not the only factors that contribute to the proper functionality of a given organism. Organisms need many more things, such as food or the presence of certain aspects of the physical environment, i.e. oxygen or surroundings of a suitable temperature. However, it is not generally assumed that these things are parts of the organism. Why not? Let us address this issue, since this seems necessary in order to properly evaluate the impact of microbiomics on the concept in question. According to the developmental definition, an organism is a network of functionally integrated cells linked by common descent. It can be understood as a consortium consisting of elements that cooperate with one another in order to keep the structure and functionality of the whole intact. Every function of such a whole is carried out by cells derived from a common recent ancestor. The whole moves because some of these cells have developed into muscle cells; it processes information from the environment because other cells have become nervous cells; it digests food because still other cells have formed a digestive tract. Thus, its cells interact dynamically to keep the organism in good shape. However, these cells do not work as a perpetuum mobile. In order to carry out such diverse and complex functions, they require certain external resources, such as food to provide energy or the proper temperature to obtain suitable conditions for the development of certain tissues. The environment obviously contributes to the functionality of the organism, but this contribution is rather passive. Elements of the environment provide necessary ingredients for the organism's cells; however, all of the organism's functions are still carried out by cells linked by common descent (e.g. through being derived from a zygote), which merely need 'fuel' from outside. This statement makes the second foundation of the developmental concept more explicit: there is a very clear distinction between the organism and its environment. The former is a set of cells linked by common descent and actively engaged in the maintenance of the organism's function and structure, whereas the latter is composed of all the remaining factors capable of influencing the functionality

\footnotetext{
${ }^{2}$ It is worth noting that this is not the only possible interpretation; one might defend a different one, starting from the same observations. For example, Janzen (1977) acknowledged the superior status of sexual over other means of reproduction, arguing that the fertilisation of an egg is an organism's real starting point; moreover, he did not regard functional integration as essential.
} 
of the organism's cells, from food and temperature to the presence of toxic proteins in surrounding waters.

Now, how can we determine whether research on microbiomes is having a revolutionary impact on this specific notion of an organism? In other words, what would prompt a scientist to think that it's about time to reject this idea and develop a new one? In the context of what has already been said, it is becoming clear that we need some new data, some premises demonstrating that the line between organisms and their environment is not sharp, but rather fuzzy, thus forcing scientists to think of organisms in a more holistic way. One way to test whether this is true in the context of microbiomics is to try to understand the nature of this kind of research. However, to make our argument even more convincing and to provide us with an element of contrast, we will first show why recent discoveries of genetic diversity within multicellular organisms, though shocking and revolutionary, do not themselves necessary suggest that we need to revise the developmental concept of the organism.

For many years, the bodies of multicellular organisms were typically thought of as genetically uniform. This view derived from a simple fact about multicellular organisms: as species such as cats or humans develop from a zygote through mitotic divisions, they should be genetically identical, since, during the process of mitosis, a cell is supposed to transmit all of its genetic material to a descendant cell. Thus, theoretically, cells of organisms that develop from a zygote should be considered genetic clones, and phenomena such as cellular mutations-such as cancer or other diseases-should be considered aberrations that disrupt the normal, uniform genetic structure of organisms. However, recent progress in science has shown that genetic diversity within organisms should be considered not as a negative exception that causes diseases, but rather as a common part of their development (Westra et al. 2010; Frank 2014). For instance, many human neurons are aneuploid; that is, they are prone to chromosomal abnormalities that lead to breaks, losses, and duplications of genetic material (Westra et al. 2010). These discoveries are revolutionising our understanding of genetic diversity within organisms - but do they force a biologist using the developmental concept of the organism outlined above to reject it? We are inclined to say 'no'. All a biologist need do in such a case is simply argue that the descendants of a zygote cell may mutate and generate diversity within an organism, because this concept does not assume that an organism must be characterised by a specific degree of genetic diversity. Therefore, these discoveries do not undermine the basic assumption of the developmental concept, i.e. that the organism is a functional whole linked by a common descent and that there is a sharp distinction between an organism and its environment.

Now, let's move on to what really interests us: the influence of microbiomics on the concept being discussed. We can picture this by using an example to make the distinction between microbiomics and discoveries such as the one described above clearer and more vivid. In the developmental concept of the organism, things like the digestive system of macrobes result from successive divisions of cells linked by common descent; these cells undergo diversification, leading to the emergence of different types of tissue belonging to various systems (nervous, digestive, etc.). For example, in cats, these cells derive from a zygote, from which a multicellular organism starts to 'bloom'. In this view, then, the zygote contains everything necessary to form a functional digestive system in a cat, a system enabling the cat to consume and assimilate food. In other words, the cells that build up a cat's digestive system are descendants of the zygote. Other cells found within the cat's digestive system are part of its environment: they either constitute the food that is being digested, or derive from some pathogens that have infected the organism. 
Microbiomics, however, makes the situation much more complex. The more we know about symbiotic microorganisms, the clearer it becomes that they are necessary for the proper functioning of the digestive systems of many species of multicellular organisms. One example is the rumen of a cow (Dehority 2003). The rumen, part of a cow's digestive system, is very important for its proper functioning, because it is where cellulose-containing material (e.g. grass) is broken down into subunits of glucose that the cow can then assimilate. This is possible thanks to microorganisms, maintained by cows in their rumens, which produce an enzyme, cellulase, which enables them to break down cellulose. Another similar example, though not as spectacular, comes from the species most familiar to us, Homo sapiens. Though it may seem that our digestive system needs no microbial support, since our diet includes very little cellulose-containing material (generally, we do not eat grass), it seems that microbes are still extremely useful for the proper functionality of our digestive system. For instance, without gut flora, we would not be able to utilise certain polysaccharides such as fibres or starch, because normally these are dealt with by certain microorganisms within the gut that produce specific enzymes (Clarke et al. 2014).

Now the question is: are these discoveries enough to force the ordinary biologist using the developmental concept of the organism to seriously consider rejecting it? We believe there are reasons to think so. To answer this question, and for the sake of clarity, suppose now that we have a macrobe that develops from a fertilised egg and interacts with symbiotic microorganisms that perform a variety of different functions. Are these microorganisms, as we said before, merely 'fuel', on a par with food or oxygen? The answer is obviously not simple, and moreover would very likely vary for different symbiotic microbes, since their roles might be extremely divergent (Douglas and Werren 2016; Queller and Strasmann 2016). Perhaps for some of them it would be justified to say that their role is similar to environmental factors (although this is debatable), because their presence may provide a certain stimulus (similar to a reduced level of oxygen, high temperature, etc.) and therefore activate certain functions of the organism's cells. However, this is not always the case. What we want to say here is that at times the difference between microbes and environmental factors may be quite substantial. There are various circumstances in which microbes may perform functions necessary for the maintenance of the structure and the functionality of the whole, functions which cannot be performed by the macrobe's cells alone. The most frequently cited examples concern digestive mechanisms, which in many species can be carried out only with the assistance of suitable microorganisms. For instance, we know that cows cannot process food containing cellulose without enzymes produced by microorganisms. Thus, microbes in such scenarios play an essentially active role ${ }^{3}$ similar to that played by a macrobe's cells derived from a zygote. These microbes are responsible for processing and assimilating external resources, and

\footnotetext{
${ }^{3}$ Microorganisms are not, of course, the only agents that may actively contribute to the maintenance and functionality of macrobes. Macrobes may do this for each other as well. For instance, bees actively contribute to the functionality of plants and vice versa. This raises a question: what is the difference between macrobe-microbes and macrobe-macrobe interactions? We think that difference is rather qualitative than quantitative. For example, since microbes inhabit a macrobe's gut, they interact continuously with the macrobe, whereas macrobes may interact in a more discontinuous way with other macrobes; they may meet to exchange some goods, and then stop interacting for a while. This is, of course, just a speculative example to show that, in our opinion, the differences are rather of a qualitative nature. Understanding the differences and similarities between these two types of interactions is very interesting, but unfortunately beyond the scope of this paper, as we are interested in analysing how macrobe-microbe interactions influence our understanding of organisms. Javier Suárez, in his paper 'Framing the Holobiont: A Framework for the Hologenome Concept of Evolution' (under review), undertakes this task, i.e. an attempt to understand and identify the differences between macrobe-microbe and macrobe-macrobe interactions.
} 
thus contribute to the undisturbed existence of the whole organism over time. Indeed, while muscle cells are responsible for movement and thereby enable such a unit to look for food, microbes may take part-along with macrobes' digestive cells-in the digestive process. Their role seems far more similar to that of the macrobe's zygote-derived cells than to that of external resources (e.g. oxygen, temperature). They are not just simply 'stuff from the outside', processed and assimilated by the macrobe's cells, but rather work together with the macrobe's cells to process and assimilate 'stuff from the outside', and thus enable the whole to endure over time. This, in our opinion, shows that they should be considered part of the organism rather than of its environment.

We believe that this, in turn, contradicts both foundations of the developmental concept of the organism outlined earlier. First, it contradicts the primary assumption of the developmental concept: that an organism is something that comes entirely from within, for example, from a zygote. The truth is that, for many species of multicellular organisms, something external, such as particular microbial cells from the environment, is also needed to perform certain tasks (e.g. digestion). It should not surprise us that the developmental concept has got it wrong by stating that cells linked by common descent (e.g. through diversification of cells) alone are sufficient. After all, this concept was developed a long time before the emergence of microbiomics; it might be attributed to Huxley (1852, pp. 187-88), who wrote: 'The individual animal is the sum of the phenomena presented by a single life: in other words, it is all those forms which proceed from a single egg, taken together'. Thus, it was impossible for those working within this paradigm to assume that something external was needed, since the majority of empirical research had shown that cats, apes, and dogs maintained their structure over time thanks to the active work carried out by their zygote-derived cells. Furthermore, the fact that multicellular organisms must incorporate objects from the environment contradicts the second assumption of the developmental concept: mainly, that a sharp distinction exists between an organism and its environment. In fact, the line is not drawn as sharply as we might think, since organisms of many species achieve their full functionality not only through the diversification of cells linked by common recent descent, but also through the acquisition of certain microbes from their surroundings. We therefore agree with scholars (see Pradeu 2011; Gilbert et al. 2012) who argue that this concept of the organism is called into question by microbiomics.

\subsection{The Cooperation-Conflict Concept of the Organism}

The second concept we want to analyse is the concept of the organism developed by Queller and Strasmann (2009). Basically, the authors argue that organisms should be understood according to just two criteria: degree of cooperation and degree of conflict among interacting elements. Thus, an organism is, in their view, a functional system built of elements that cooperate to sustain its stability. Accordingly, the greater the degree of cooperation and the lesser of conflict between elements of such a system, the greater the organismality of the unit. To be an organism is thus, in light of the varying degrees of cooperation and conflict among species in nature, a continuous variable. Indeed, some combinations of units are highly organismal, because they are characterised by a high degree of cooperation and a low degree of conflict: for example, a cat, built of cooperating cells, might be described in this way. Alternatively, other combinations of units are not organismal at all, as they are characterised only by a high level of conflict, the most spectacular example being interactions between hosts and parasites.

Now let us ask: what is the most important part of this conceptualisation? It should be clear that the core of this cooperation-conflict concept of the organism is as follows: to be 
an organism, a group of elements must be characterised by a high degree of cooperation and a low level of conflict. But what do these terms really mean? According to the general definition, cooperation refers to the possession of mechanisms that make a group of elements a cohesive, functional whole. On the other hand, the term low level of conflict refers to the possession of mechanisms which reduce potential conflict among elements in an organism to a minimum. A conflict arises when an element within a group does not behave so as to promote the functionality of the whole, but rather acts in a selfish manner, to the detriment of the whole. Therefore, conflict-suppressing mechanisms are necessary in order to exist as a functional and cohesive unit. To picture what these two kinds of mechanisms look like in practice, let us provide an example: a cat, which we considered earlier to be characterised by a high level of cooperation and a low level of conflict, making it a paradigm of an organism in the evolutionary framework.

When we look at the body of a cat, we immediately realise that it is a group of cells characterised by a high level of cooperation, to the extent that we intuitively consider it to be a single unit (just as we do with other animals, e.g. apes or cows). This is because cats belong to an evolutionary line that, in the course of evolution, has developed sophisticated cooperative mechanisms which have transformed these cats into cohesive, functional wholes. We find here cells of the digestive system responsible for the cat's digestion of food, muscle cells responsible for the cat's movement, as well as nervous cells that enable the cat to respond to environmental stimuli. Thus the group of cells making up the cat is a highly cooperative union adapted to work as a functional whole. Nevertheless, there may still be some room for the emergence of internal conflict. One example is cancer, which is not limited to cats or animals, but which seems to be a kind of conflict common to all multicellular organisms (see Aktipis et al. 2015). Basically, by cancer we mean a situation in which a cell stops performing its normal functions (mainly due to mutations) and starts to reproduce, at an uncontrolled rate, to the detriment of the organism, leading very often to its death. However, the evolutionary line to which cats belong has developed mechanisms that minimise the potential of such conflict. One such mechanism is a general division of labour among cells that goes even further than the one mentioned above: division into somatic and germ-line cells (Michod and Roze 1999). The former are responsible for the cat's survival, the latter for its reproduction. This sequestration reduces the potential for conflict, because it reduces the chance of mutated somatic cells being transmitted to the next generation. Thus it minimises the chance that cancer will emerge in the next generation (the more mutations a cell undergoes, the more likely it is that it will be transformed into a cancer cell, although mutations alone are not always sufficient for cancer to emerge, as there are other essential factors in play).

Now let us turn to microbiomics. Would the discoveries of microbiomics force a biologist using this cooperation-conflict concept of organism to think of rejecting it? We do not believe that microbiomics undermines the foundations of this conceptualisation, which is flexible enough to permit us to think of a host and its symbiotic microorganisms as a functional whole-an organism. This is true because this concept, rather than focusing on any kind of phenotypic trait which would indicate organismality (such as common descent, genetic uniqueness, etc.), instead uses the abstract terms cooperation and conflict, which can be implemented differently by different units. Therefore, if a given combination of a host and its symbiotic microorganisms fulfils the criteria of a high level of cooperation and a low level of conflict, we can consider this combination to be an organism, because it has evolved to the extent that its elements work in a coordinated way, and, furthermore, thanks to some kind of adaptation, conflict between its elements has been minimalised. Is this true in the case of interactions between symbiotic microorganisms and multicellular organisms? 
Queller and Strasmann (2016) argue that some host-microorganism combinations have developed such a high level of cooperation and a low level of conflict that it would be rational to consider them as a kind of multi-species organism. One such case is exemplified by aphids and their symbiotic bacteria Buchnera sp. (Baumann 2005). These bacteria are not picked up from the environment, but are transmitted between aphid generations through special propagules called bacteriocytes during early development. Therefore, the relationship has reached the point that the two parties have lost their autonomy and become one complex unit; together, they function in a coordinated, interdependent way. The bacteria obtain protection and, in exchange, synthesise the amino acids lacking in the aphids' diet. Besides a high level of cooperation, other things exist as well which reduce conflict between the elements of this combination to a minimum. One such thing, among many, is drastic reduction of the bacterial genome (McCutcheon and Moran 2012). Many genes have been lost in the course of the bacteria's evolution, which means that fewer genes can be brought to bear in conflicts (the existence of fewer genes equates to a reduced probability that one of them will mutate and harm the aphid). Thus, it makes sense to say that such a combination scores high on the scale of organismality.

Unfortunately, as one might suppose, not all interactions between multicellular organisms and symbiotic microorganisms would do as well on the scale of organismality. For instance, certain bacterial pathogens currently subject to intensive medical studies, such as Helicobacter pylori or Vibrio cholerae (see Pan et al. 2014), have a detrimental impact on the human body; thus, in the case of such interactions, there is not much cooperation and a great deal of conflict. However, between pathogens and well-suited combinations such as aphids and Buchnera are numerous intermediate states of organismality, since the whole concept is not absolute but measured by degrees; the most obvious cases (cats, apes and so on) evolved from organisms that, perhaps millions of years ago, were characterised by a very low (or average) level of cohesion and interdependence among elements. Currently, it is likely that many interactions between microbes and multicellular organisms represent such an intermediate level of organismality. For instance, in the case of symbiotic bacteria living within the human gut, we indeed benefit from some kind of cooperation, because our (the host's) immune system tolerates them and they are engaged in the functioning of our immune and digestive system (Ley et al. 2008). However, there is some room for conflict between them as well, because these relationships are not as fixed as in the case of aphids and Buchnera; for instance, the diet of the host might change the composition and abundance of symbiotic microbes (David et al. 2014); what is more, the majority of symbiotic microbes could live just as well outside the host (Ley et al. 2006, 2008). Thus, as their reproductive successes are not very strongly linked, one might expect that if some factors were to change, some microbes might, for instance, 'decide' to reproduce faster at the expense of the host. This should not surprise us, as similar shifts from cooperation to defection occur quite often in nature as factors such as availability of resources change (Hoek et al. 2016).

The cooperation-conflict concept of the organism, in the context of the preceding considerations, demonstrates the very complex nature of holobionts. Holobionts are not unified objects, because they exhibit varying levels of cooperation and conflict between hosts and microbes. Indeed, as we showed in the last few paragraphs, only certain combinations of host and symbiotic microbes are characterised by such a high level of cooperation and low level of conflict that we can consider them examples of proper organismality. Therefore, in the context of the cooperation-conflict concept of the organism, it is not really justified to argue that a host and all of its symbiotic microorganisms constitute a good example of a highly organismal entity. Rather, this can be said 
about only a very small number of symbiotic microbes. This raises a number of interesting - both philosophically and scientifically-questions. What factors favour cooperation between hosts and microbes? Through what physiological mechanisms is cooperation between microbes and hosts sustained? Are these mechanisms the same across all species? How are conflicts of interest resolved? These and many other questions will be definitely addressed in the next years by scholars, since they appear to be necessary in order to gain a deeper understanding of the factors that make a host and microbes an organism in the context of the cooperation-conflict concept of the organism.

The cooperation-conflict concept of organism seem to cope well with the ongoing discoveries of microbiomics. Indeed, it seems that these discoveries are not at all revolutionary from the perspective of this (as opposed to the developmental) conceptualisation. Moreover, the assumptions of this theory enable us to easily incorporate these recent discoveries. Scientists working within this framework would say that what is really going on right now is simply a gradual accumulation of new data-data that are expanding our understanding of elements that might group together to form cooperative unions. Interestingly, these may include not only cells from the division of zygotes, but also bacterial cells from the environment that can be incorporated into organisms. Indeed, from the perspective of this conceptualisation of the organism, the discoveries of microbiomics demonstrate new ways of interacting among cooperative elements without undermining the foundations of the whole idea. Thus, for people working within this framework, it is far from true that their understanding of the organism is changing. There are only ordinary discoveries of new interactions among elements that constitute organisms.

\section{Making Sense of the Results}

Let us summarise some of the main points we made in the last section. Biologists have not developed a concept of the organism that would be universally accepted; rather, within biology, alternative definitions exist. Furthermore, the differences between various conceptualisations are not merely cosmetic, but (as we have shown based on two analysed concepts) fundamental. Specifically, these differences influence the way a given conceptualisation deals with current discoveries in the field of microbiomics. Some concepts which, for certain reasons, are unable to explain and incorporate the results of the ongoing discoveries of microbiomics must be rejected, while others are flexible enough to do so quite easily. This assures us that the current impact of microbiomics has a local character. Only some conceptualisations of the organism must be rejected.

So far we have argued that microbiomics has an irregular impact on the understanding of organisms across biology. However, this is only a raw analysis of the issue. To get a more comprehensive picture of the influence of microbiomics on the concept of the organism, we have to take a closer look into its nature. There are two basic questions that should be asked in order to make this clear. Firstly, why there are so many co-existing concepts of the organism? Secondly, can answering this question help to explain why some concepts deal with microbiomics better than others?

Why are there so many concepts of the organism? The very basic answer to this question is that living things are very complex and it would be hard to develop a concept of the organism applicable to different objects, such as social insects, humans, bacteria, plants, and fungi. This is because, despite a consensus among scientists that an organism is a kind of functional, cohesive whole (Pepper and Herron 2008; Queller and Strasmann 
2009; Clarke 2010), when it comes to setting boundaries between organisms in nature the issue becomes much more complex. It is not so easy to choose criteria that would fit all species. Therefore, one might think that what really has to be done to solve this problem is to elaborate on the concept of the organism, resulting in the development of one that would be general enough to be used by scientists to study different taxa. Indeed, many such attempts have been recently put forward (see Korzeniewski 2001; Queller and Strasmann 2009; Clarke 2013).

However, there is a basic problem with this approach: mainly, that it presupposes only one proper way of picking out individuals in nature. Indeed, following the logic of the previous paragraph, one might easily reach the conclusion that there is one universal concept of the organism waiting to be discovered by a prominent philosopher or scientist and thus we just have to wait until someone develops it. But is it really true that there is only one 'right' way to differentiate individuals? In other words, can scientists favour one way of individuating objects over others based on some sort of rational choice? If true, this would mean that we should prioritise one approach which somehow does a better job of capturing the 'essence' of being an individual. An obvious candidate for this would be the theory of evolution by natural selection, which is the theory that unifies biology.

Recently many scholars have defended a different position, one which we think explains the existence of multiple conceptualisations of the organism in an elegant manner. Namely, they have suggested that the priority of a single concept need not necessarily apply within biology (see Pepper and Herron 2008; Dupré and O'Malley 2009; Kovaka 2015; Pradeu 2016). As an alternative, a pluralistic approach might be a good way to individuate organisms, since there may be more than one way to single out individuals. Scientists might choose to use a concept of the organism based on their research aims. In other words, when scientists change the aim of their research, they may also change their criteria for individualisation-which basically means that what dictates the individualisation of objects by biologists is scientific practice. Let's consider some examples in order to make this much clearer.

It would not be controversial to say that the developmental concept of the organism is very popular in the study of development of animals (Slack 2005, p. 6; Pradeu 2011; Moore et al. 2016, p. 1). The explanation for this is quite obvious. Generally, developmental biologists studying animals are interested in understanding how such complex things as an eye, a digestive system, etc., develop from a fertilised egg. To perform this task, they follow the paths of cells undergoing division and their diversification and try to identify the factors responsible for this, manipulating external factors such as salinity, temperature, diet, etc. in order to observe how they influence the development of particular animals. Therefore, the application of the developmental concept appears to be a really good choice for this kind of research, since it is congruent with scientific practice conducted within the field of developmental biology. On one hand we have a concrete, defined organism which is the object of our inquiry: a set of interacting cells that arises as an effect of the successive divisions of a zygote. On the other hand, we have external elements which we can manipulate to monitor changes in development of organisms.

This concept, however, becomes problematic when we try to use it for other purposes. For example, if we are interested in research concerning species as different as flowers, aphids, and bacteria at the same time, then perhaps a more abstract concept of the organism is appropriate, such as the cooperation-conflict concept of the organism (Queller and Strasmann 2009, 2016; Strassmann and Queller 2010), since this concept is general enough to be easily applied to different species. This feature makes the concept very useful, for instance, in comparative studies between species, as it provides an abstract, theoretical 
framework within which to organise such an interspecies analysis. For instance, if we want to understand whether biofilms (packed communities of bacterial cells) are as well integrated as multicellular organisms, we might ask what level of cooperation and conflict characterises the former compared to the latter. This might help us to understand the differences and similarities between them and, therefore, to draw more justified conclusions.

However, both of these concepts might prove useless for other scientific aims. For instance, for a palaeontologist working in the field, it is more comfortable to focus on anatomical indicators of individuality (such as bones) than on aspects such as common descent or abstract terms of cooperation and conflict. Indeed, when a palaeontologist deals with bones, it is not always known whether his or her object of study developed from a fertilised egg, or whether it possessed effective mechanisms of suppressing internal conflicts; thus it would be hard to apply one of the above-mentioned concepts here in order to single out individuals from the remains of organisms. This kind of scientist would prefer to use some kind of anatomical concept, one which considers an organism as an anatomical whole. According to such an approach, a set of bones would constitute one organism if it makes up, from an anatomical point of view, a functional and cohesive whole. For example, a palaeontologist would link 'tibia A' and 'femur B' if he or she saw that both were capable of interacting in a functional manner, thus suggesting that they are part of the same whole. Therefore, a set of bones would be called an organism not because of some observable mechanism, but because the bones visibly seem to make up an anatomically functional whole.

These examples show that what actually determines the choice of a concept of the organism is its usefulness in certain research procedures. This should not seem surprising if we look at the way biological sciences are organised. Biology is an extremely decentralised science, encompassing many different subfields. Scientists working in these areas of research have often developed their own procedures, created their own conferences, journals, etc.; this mirrors the fact that they are interested in studying different aspects of the biological world. This diversity of scientific goals often requires different tools and methodologies, something that obviously influences the way particular objects of inquiry are conceptualised. This can be clearly observed in the case of concepts of the organism, as we have shown in the last few paragraphs. For different scientific aims, biologists tend to define organisms in different ways, depending on the usefulness of the definition in comparison to other concepts. Thus, it seems that the co-existence of so many different concepts of the organism in biology is caused by the fact that scientists may conceptualise individuals in different ways for different purposes.

Can this help us explain why microbiomics is making such an irregular impact on the concepts of the organism used by biologists? In other words, can this help us explain why some concepts can deal with discoveries of microbiomics, while others cannot? We think so. This will become clear the moment we realise what it really means when we say that scientific practice dictates the choice of the concept of the organism. Different scientific purposes require different concepts, because scientists do different things, and thus can make different assumptions about organisms. If we study just one species, for example cats, we do not need to define organisms in a way that captures organismality in, let's say, biofilms. Indeed, while conducting our cat-based research we are not really interested in other species; therefore we can define organisms very narrowly, for example, by referring to more concrete traits that are good indicators of organismality in this species (such as common descent, genetics uniqueness, etc.). However, if we are conducting a comparative study covering many different species, we'll use a concept of the organism that can be 
applied to all of them. Such studies require a more abstract, general concept, such as cooperation-conflict, because we want to apply it to many different species. Therefore, using ideas of a general nature is not always necessary. Rather, it seems that in some cases it is justified to use a narrow concept, while in others it is necessary to assume a broader, more general idea, if it is more suitable for the purposes of particular research.

That scientists sometimes define organisms more broadly and other times more narrowly is, in our opinion, the main reason for the local character of the influence of microbiomics on the concepts of the organism. Why do we think so? Because we believe that narrowly-defined concepts (e.g. those referring to more concrete things, such as common descent or genetic uniqueness) are more likely to be rejected when new discoveries emerge. This is because they are based on more concrete, area-dependent assumptions and thus it is more likely that new discoveries will prove them wrong. On the other hand, concepts based on more abstract terms are more likely to be immune to novel discoveries, as they are more independent of particular research areas. Although it may turn out eventually that part of our knowledge about organisms is misguided, this will not necessary lead to the rejection of such a concept, since new discoveries may also indicate that the functionality of organisms is carried out in different ways. This explains why, for the purposes of this paper, we have chosen the developmental and the cooperation-conflict concepts of the organism: the first is defined in a narrow, a more concrete way, the second one in a broader and more abstract manner. We suspect that, due to this significant dissimilarity, they will respond differently to ongoing discoveries and thus show that the current influence of microbiomics on concepts of the organism has a local character.

This, actually, is what we have shown in the previous section. The developmental concept was unable to deal with microbiomic research, while the cooperation concept of organism had no problems with it. This was because the assumptions on which the former is based are more concrete, while those of the latter are based on abstract terms of cooperation and conflict; microbiomics merely introduces new kinds of co-operative interactions among elements. Even though we have analysed only two concepts, there are good reasons to think that the same irregularity may concern other concepts of the organism. This is because the developmental concept is not the only one that rests on specific assumptions; there are others that make such assumptions (examples include genetic structure and spatial boundaries), and research on microbiomics might force us to revise them. Other concepts, however, may be broad enough to deal with microbiomics (see Dupré and O'Malley 2009; Godfrey-Smith 2013; Moran and Sloan 2015; Douglas and Werren 2016; Pradeu 2016). For instance, Dupré and O'Malley (2009) use the concept of the interactor to show how to properly understand interactions between macrobes and microbes. Therefore, they have shown that the existing concepts of individuals are broad and flexible enough to be used to understand the results of research on microbiomics.

Bearing in mind this specific nature of the concept of the organism within biology, we can now fully understand why microbiomics has a local influence on the concepts of organism. Biology is a very broad science, and researchers choose various research subjects, from the biodiversity of tropical forests to the diversity of cat populations to the very nature of DNA replication. For some of these scientific aims, narrow conceptualisations of the organism are sufficient. For example, if one studies the development of cats, then the developmental conceptualisation of the organism would be a good choice: it enables scientists to distinguish one cat from another and is easy to apply in the laboratory. For other scientific problems, however, a more abstract concept would be better, especially for someone interested in studying many different species. Thus biology contains many different conceptualisations of organisms. Research on microbiomes, while spectacular, 
makes such an impact on certain concepts that the scientists using them are forced to revise them. Those concepts which are more likely to experience the impact of microbiomes are those defined in a very narrow, concrete way, because they are based on more concrete assumptions and new discoveries are more likely to invalidate those assumptions. This is, perhaps, what researchers studying the development of animals have to do, because it seems that understanding the development of their object of study is impossible without taking symbiotic microbes into consideration and thus shifting from the developmental concept of the organism to a more holistic definition (see Pradeu 2011). On the other hand, many other scientists would regard research on microbiomes as an interesting nuance and would not feel impelled to revise their concept of the organism, because the concept they use is broad enough to incorporate the results of research on microbiomes.

This raises an interesting question. Will the fact that some concepts can cope better with the discoveries of microbiomics lead to a situation where scientists switch en masse to more flexible concepts? For example, we showed that the co-operation-conflict concept can incorporate the latest results of microbiomic research, whereas the developmental concept of organism is incapable of doing so. Does it mean that scientists will now, after reading our paper, switch to the new concept? It may seem that way. As we said in the last paragraph, those using the developmental concept of the organism may need a more holistic one, and the co-operation-conflict concept looks like a good candidate. We think, though, that this is very unlikely. If a scientist has studied some aspects of human biology using the developmental concept of the organism and now, for some reason, must incorporate microbiomics into his research and consequently reject the developmental approach, it is unlikely that he'll switch to the co-operation-conflict concept of organism. This concept is so broad that it enables us to speak about the organismality of plants and bees (Queller and Strasmann 2009, 2016; Strassmann and Queller 2010); however, our scientist has no need of such an idea. Rather, he simply needs to understand the place of microbes in the case of individuality of humans. Thus, he will very likely develop a concept that will transcend the developmental one by simply adding information on microbes, one that will suit his research process very well, as nicely expressed by Sleator (2010) at the beginning of this paper: "For years our traditional view of 'self' was restricted to our own bodies, composed of eukaryote cells encoded by our genome. However, in the era of omic technologies and systems biology, this view now extends beyond the traditional limitations of our own core being to include our resident microbial communities." This shows, in our opinion, that the 'microbiomic revolution' does not lead to conceptual unity, i.e. is not a step toward a unified concept of the organism. The "organismal landmark" present within biology will not change. Prior to the emergence of microbiomics, many concepts of the organism existed, and after microbiomics many will continue to exist. Only certain scientists will have to reformulate the concept they use. This should not be controversial. After all, if the concept of the individual depends on scientific practice and there is no unity of practice within biology, why should we expect unity in conceptualisation?

\section{Concluding Remarks}

Understanding how research on microbiomics is changing biology has recently been one of the topics in biology most frequently debated by both philosophers and biologists, especially the analysis of the concept of the organism (Dupré and O'Malley 2009; Gilbert et al. 2012; Pradeu 2016). In this paper we have presented a novel perspective on this subject, 
according to which the various conceptualisations respond differently to ongoing discoveries; thus, change in the understanding of organisms in biology is local, constrained to certain concepts of the organism which cannot manage to incorporate the ongoing discoveries. This does not, of course, diminish the outstanding discoveries of microbiomics. Rather, it shows that biology as a scientific field is very complex and divided into many subfields; scientists working within one field might conceptualise their objects of inquiry in a different manner than those working within another. Nor does this concern only the concept of the organism, as conceptual pluralism applies as well to other concepts used by scientists, e.g. species (Dupré 1999; Suárez 2015) or population (Stegenga 2016). Therefore, it is very likely that microbiomics will influence each variant of such a concept in a different way and thus lead as well to local changes. But is conceptual pluralism applied universally? In other words, does this mean that biology is the kind of science that always permits its practitioners to have many alternative definitions of a given concept? We do not think that this is in fact true. For instance, within biology there is only one concept of DNA (Brown 2006), and its rejection would very likely lead to rearrangement of the entire field. Therefore, it is very possible that research on microbiomics will make different impacts on different concepts in biology. For example, sometimes it may lead to local changes; other times, if it undermines a concept that is very well grounded within all subfields of biology, it may lead to complete rearrangement. Our contribution to understanding the influence of microbiomics was to show that, at least in the case of concepts of the organism, we are dealing with local changes. We hope that this new perspective will enrich our understanding of the current transformation of biology by microbiomics. Furthermore, we hope that it will also enhance the mutual understanding of scientists working in different fields. The fact that novel discoveries seem not to challenge the basic concepts of a given scientist does not necessarily mean that they will not challenge those of other scientists, who may individuate things like organisms, genes, populations, species, etc., in a different way and thus feel the impact of novel discoveries in a different manner.

Acknowledgements We thank Jerzy Gołosz and Josef Ulatowski for their useful remarks on the earlier versions of the paper as well as the two anonymous reviewers for their careful reading of our manuscript and their insightful comments and suggestions. We thank also Javier Suárez and John Dupré for the fruitful discussions which greatly contributed to improving the final version of the paper. The authors conceived and wrote the paper together. This work was financed by Jagiellonian University Internal Grant (K/DSC/003898) to A.S. and by Jagiellonian University Internal Grant (K/DSC/003897) to A.P.

\section{Compliance with the Ethical Standards}

Conflict of interest The authors declare that they have no conflict of interest.

Open Access This article is distributed under the terms of the Creative Commons Attribution 4.0 International License (http://creativecommons.org/licenses/by/4.0/), which permits unrestricted use, distribution, and reproduction in any medium, provided you give appropriate credit to the original author(s) and the source, provide a link to the Creative Commons license, and indicate if changes were made.

\section{References}

Aktipis, C., Boddy, A., Jansen, G., Hibner, U., Hochberg, M., Maley, C., et al. (2015). Cancer across the tree of life: Cooperation and cheating in multicellularity. Philosophical Transactions of the Royal Society of London. Series B, Biological sciences, 370(1673), 20140219.

Baquero, F., \& Nombela, C. (2012). The microbiome as a human organ. Clinical Microbiology \& Infection, $18(4), 2-4$. 
Baumann, P. (2005). Biology of bacteriocyte-associated endosymbionts of plant sap-sucking insects. Annual Review of Microbiology, 59, 55-189.

Brown, T. (2006). Genomes 3. New York: Garland Science.

Brucker, R., \& Bordenstein, S. (2013). The hologenomic basis of speciation: Gut bacteria cause hybrid lethality in the genus Nasonia. Science, 341(6146), 667-669.

Carabotti, M., Scirocco, A., Maselli, M., \& Severia, C. (2015). The gut-brain axis: Interactions between enteric microbiota, central and enteric nervous systems. Annals of Gastroenterology, 28, 203-209.

Clarke, E. (2010). The problem of biological individuality. Biological Theory, 5(4), 312-325.

Clarke, E. (2013). The multiple realizability of biological individuals. Journal of Philosophy, 110(8), 413-443.

Clarke, G., Stilling, R., Paul, J., Kennedy, P., Stanton, C., Cryan, J., et al. (2014). Minireview: Gut microbiota: The neglected endocrine organ. Molecular Endocrinology, 28(8), 1221-1238.

David, L., Maurice, C., Carmody, R., Gootenberg, D., Button, J., Wolfe, B., et al. (2014). Diet rapidly and reproducibly alters the human gut microbiome. Nature, 505, 559-563.

Dehority, B. (2003). Rumen microbiology. Nottingham: Nottingham University Press.

Douglas, A., \& Werren, J. (2016). Holes in the hologenome: Why host-microbe symbioses are not holobionts. Bio, 7, e02099-15.

Dupré, J. (1999). On the impossibility of a monistic account of species. In R. Wilson (Ed.), Species: New interdisciplinary essays (pp. 3-22). Cambridge: MIT Press.

Dupré, J., \& O’Malley, M. A. (2009). Varieties of living things: Life at the intersection of lineage and metabolism. Philosophy \& Theory in Biology, 1, e003.

Frank, S. A. (2014). Somatic mosaicism and disease. Current Biology, 24, R577-R581.

Gilbert, S., Sapp, J., \& Tauber, A. (2012). A symbiotic view of life: We have never been individuals. The Quarterly Review of Biology, 87(4), 325-341.

Godfrey-Smith, P. (2009). Darwinian populations and natural selection. Oxford: Oxford University Press.

Godfrey-Smith, P. (2013). Darwinian individuals. In F. Bouchard \& P. Huneman (Eds.), From groups to individuals: Evolution and emerging individuality. Cambridge: MIT Press.

Hoek, T., Axelrod, K., Biancalani, T., Yurtsev, E., Liu, J., \& Gore, J. (2016). Resource availability modulates the cooperative and competitive nature of a microbial cross-feeding mutualism. PLoS Biology, 4(8), e1002540.

Huxley, T. H. (1852). Upon animal individuality. Proceedings of the Royal Institution, 1, 184-189.

Janzen, D. (1977). What are dandelions and aphids? American Naturalist, 111, 586-589.

Korzeniewski, B. (2001). Cybernetic formulation of the definition of life. Journal of Theoretical Biology, 209(3), 275-286.

Kovaka, K. (2015). Biological individuality and scientific practice. Philosophy of Science, 82(5), 1092-1103.

Lederberg, J. (2000). Infectious history. Science, 288, 287-293.

Ley, R., Lozupone, C., Hamady, M., Knight, R., \& Gordon, J. (2008). Worlds within worlds: Evolution of the vertebrate gut microbiota. Nature Review of Microbiology, 6, 776-788.

Ley, R., Peterson, D., \& Gordon, J. (2006). Ecological and evolutionary forces shaping microbial diversity in the human intestine. Cell, 124(4), 837-848.

Mazmanian, L., Liu, C., Tzianabos, A., \& Kasper, D. (2005). An immunomodulatory molecule of symbiotic bacteria directs maturation of the host immune system. Cell, 122(1), 107-118.

McCutcheon, J., \& Moran, N. (2012). Extreme genome reduction in symbiotic bacteria. Nature Review of Microbiology, 10, 13-26.

McFall-Ngai, M., Hadfield, M. G., Bosch, T. C., Carey, H. V., Domazet-Loso, T., et al. (2013). Animals in a bacterial world, a new imperative for the life sciences. Proceedings of the National Academy of Sciences of the United States of America, 110(9), 3229-3236.

Michod, R., \& Roze, D. (1999). Cooperation and conflict in the evolution of multicellularity. Heredity, 86, $1-7$.

Moore, K., Persaud, T., \& Torchia, M. (2016). Before we are born: Essentials of embryology and birth defects (9th ed.). Philadelphia, WB: Saunders Company.

Moran, N., \& Sloan, D. (2015). The hologenome Concept: Helpful or hollow? PLoS Biology, 13(12), e1002311.

O’Malley, M. A., \& Dupre, J. (2007). Size doesn't matter: Towards a more inclusive philosophy of biology. Biology and Philosophy, 22, 155-191.

Pan, X., Yang, Y., \& Zhang, J.-R. (2014). Molecular basis of host specificity in human pathogenic bacteria. Emerging Microbes \& Infections, 3, e23.

Pepper, J., \& Herron, M. (2008). Does biology need an organism concept? Biological Reviews, 83(4), $621-627$. 
Pradeu, T. (2011). A mixed self: The role of symbiosis in development. Biological Theory, 6(1), 80-88.

Pradeu, T. (2016). Organisms or biological individuals? Combining physiological and evolutionary individuality. Biology and Philosophy, 31(6), 797-817.

Queller, D., \& Strasmann, J. (2009). Beyond society: The evolution of organismality. Philosophical Transactions of the Royal Society B, B364, 3143-3155.

Queller, D., \& Strasmann, J. (2016). Problems of multi-species organisms: Endosymbionts to holobionts. Biology and Philosophy, 31(6), 855-873.

Santelices, B. (1999). How many kinds of individual are there? Trends in Ecology \& Evolution, 14, $152-155$.

Sender, R., Fuchs, S., \& Milo, R. (2016). Revised estimates for the number of human and bacteria cells in the body. PLoS Biology, 14(8), e1002533.

Sharon, G., Segal, D., Ringo, J., Hefetz, A., \& Zilber-Rosenberg, I. (2010). Commensal bacteria play a role in mating preference of Drosophila melanogaster. Proceedings of the National Academy of Sciences of the United States of America, 107(46), 20051-20056.

Slack, J. (2005). Essential developmental biology (2nd ed.). Oxford: Blackwell Publishing LTD.

Sleator, R. (2010). The human superorganism-Of microbes and men. Medical Hypotheses, 74(2), 214-215.

Stegenga, J. (2016). Population pluralism and natural selection. The British Journal for the Philosophy of Science, 67(1), 1-29.

Strassmann, J., \& Queller, D. (2010). The social organism: Congresses, parties, and committees. Evolution, $64,605-616$.

Suárez, J. (2015). Bacterial species pluralism in the light of medicine and endosymbiosis. Theoria, 31(1), 91-105.

Westra, U., Rivera, R. R., Bushman, D., Yung, Y. C., Peterson, S., Barral, S., et al. (2010). Neuronal DNA content variation (DCV) with regional and individual differences in the human brain. Journal of Comparative Neurology, 518(19), 3981-4000.

Wilson, J. A. (2000). Ontological butchery: Organism concepts and biological generalizations. Philosophy of Science, 67, S301-S311.

Zilber-Rosenberg, I., \& Rosenberg, E. (2008). Role of microorganisms in the evolution of animals and plants: The hologenome theory of evolution. FEMS Microbiology Reviews, 32(5), 723-735.

Adrian Stencel is a Ph.D. student in Philosophy and teaching assistant in the Department of Philosophy at the Jagiellonian University in Cracow, where he teaches methodology of science. He graduated from Biology at Jagiellonian University. His research interest focus on philosophy of biology, with strong interest in the conceptual and foundational issues surrounding the theory of evolution, population biology and microbiology.

Agnieszka M. Proszewska is a Ph.D. student in Philosophy and teaching assistant in the Department of Philosophy at the Jagiellonian University in Cracow, where she teaches logic and set theory, epistemology and theory of computation. She graduated from Philosophy and Swedish Philology at the Jagiellonian University and currently she is also working on her Master's thesis in theoretical computer science at the Department of Physics, Astronomy and Applied Computer Science. Her research interests focus on the philosophy of natural sciences, structural frameworks and mathematical logic. Since 2014 she serves as a regular member of Polish Artificial Intelligence Society and European Philosophy of Science Association. 